\section{Let's twist again: elasto-capillary assembly of parallel ribbons}

\section{Supplementary Information}

by A. Legrain, E.J.W. Berenschot, L. Abelmann, J. Bico and N.R. Tas
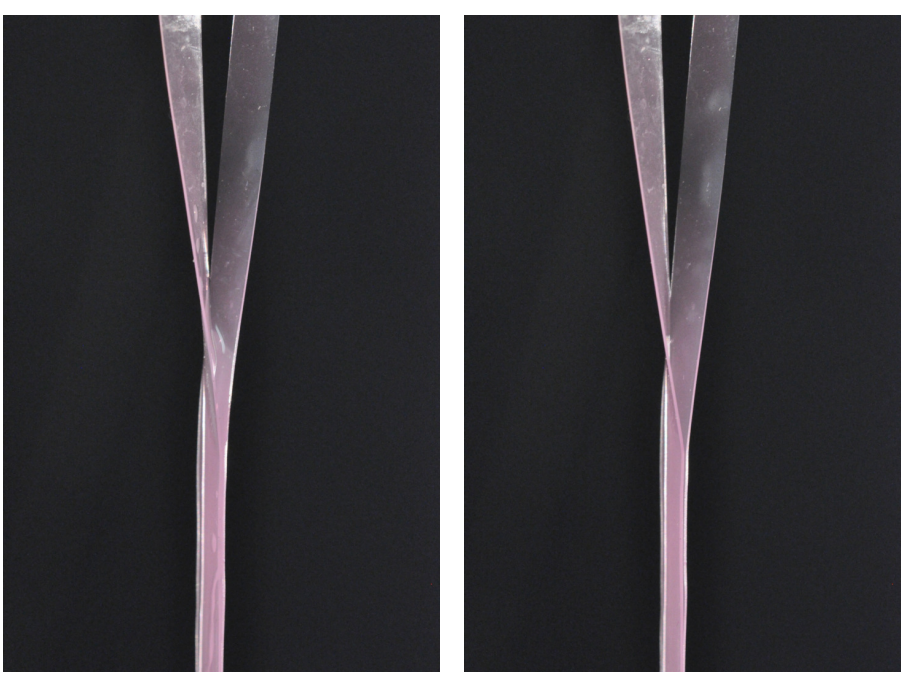

Figure S1 Close-up view of the contact region in the case of macroscopic ribbons of width $w=2 \mathrm{~mm}$. The ribbons are cut from a sheet of polyvinylsiloxane rubber (PVS) of thickness $t \sim 150 \mu \mathrm{m}$ and of Young modulus $E \sim 330 \mathrm{kPa}$.s. We used ethanol as adhering liquid. In the left image the liquid meniscus is still present. In the right image the liquid evaporated, but the ribbons remain in contact through van der Waals interactions (promoted by the high softness of the rubber). We expect similar interactions to maintain micro-ribbons in contact after evaporation of the adhering liquid. Note that the contact line between the ribbons is oblique. However, the effect of this tilt can be neglected in the estimation of the opening length in the limit $w \ll l$.
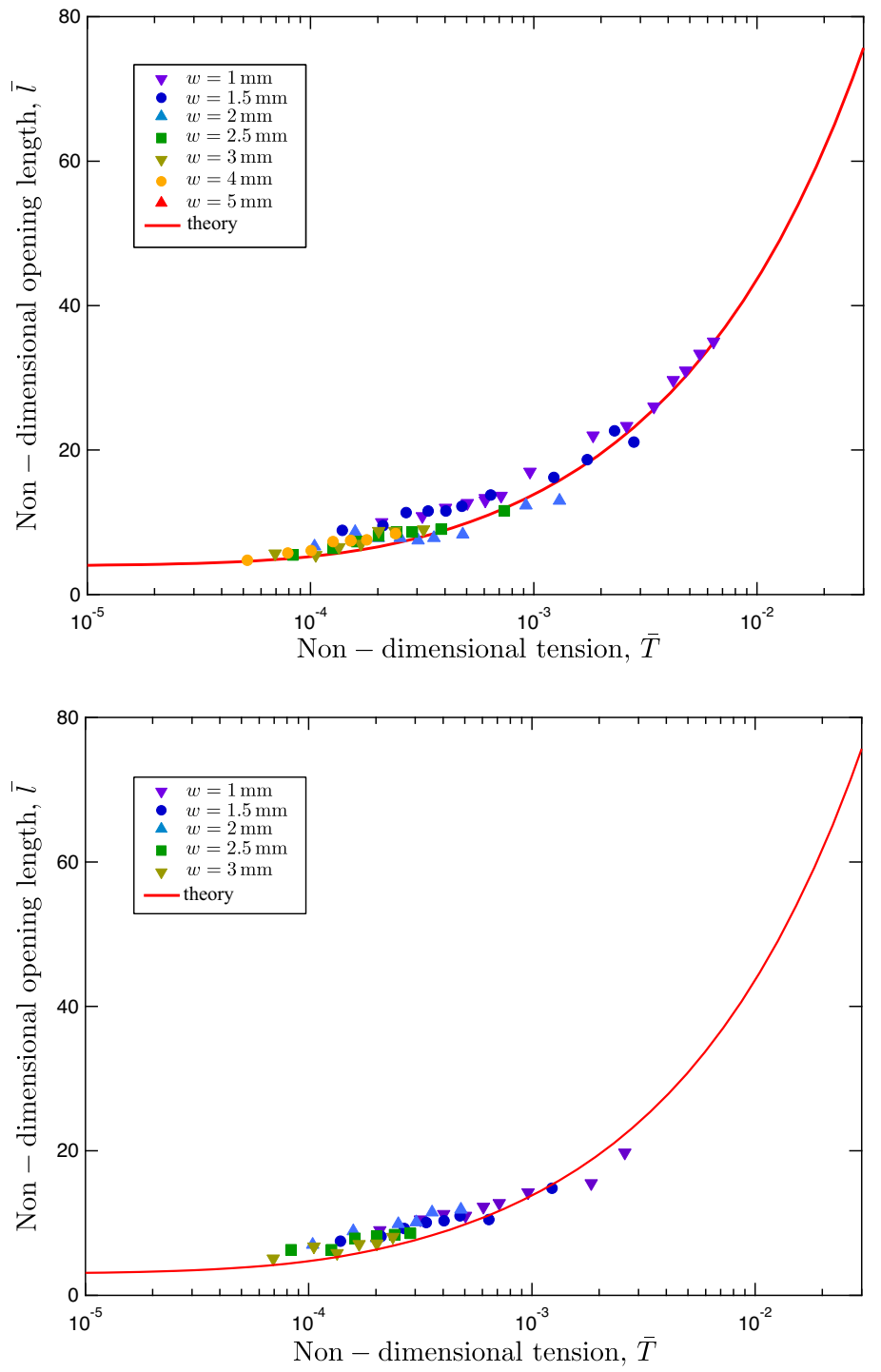

Figure S2 Normalized opening length $\bar{l}=l / d$ as a function of the normalized tension $\bar{T}=F / E h w$ for $\bar{w}=w / d=1 / 3$ (top) and $\bar{w}=w / d=1 / 4$ (bottom). The line correspond to the corresponding theoretical prediction (with $\beta=1$ ). 


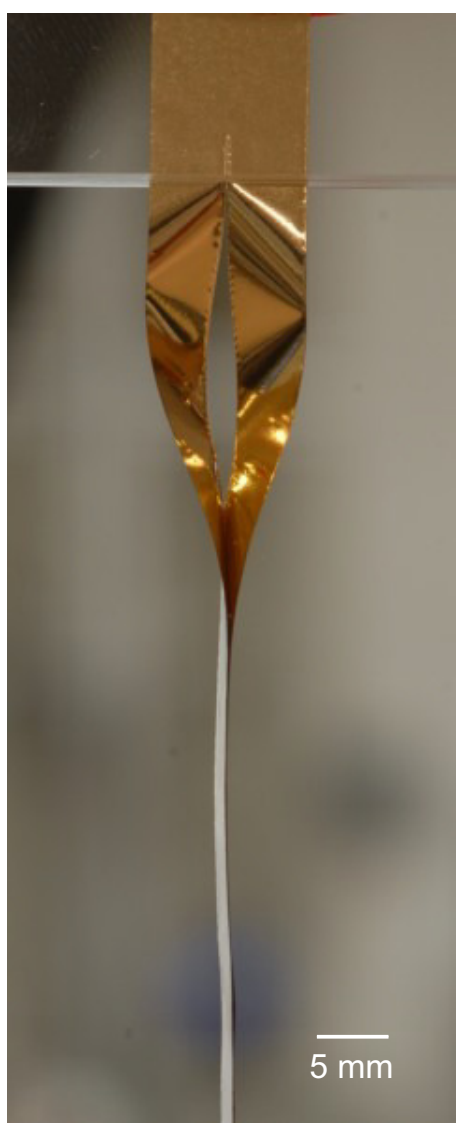

Figure S3 Macroscopic ribbons tend to form creases when for low tensions and short opening lengths. In the current experiment the normalized tension is of order $\bar{T} \simeq 10^{-4}$ and the normalized twist angle $\pi w / l \simeq 0.6$. The observation of creases is thus in perfect agreement with the recent study from Chopin and Kudrolli, Phys. Rev. Lett. (2013) 111 174302
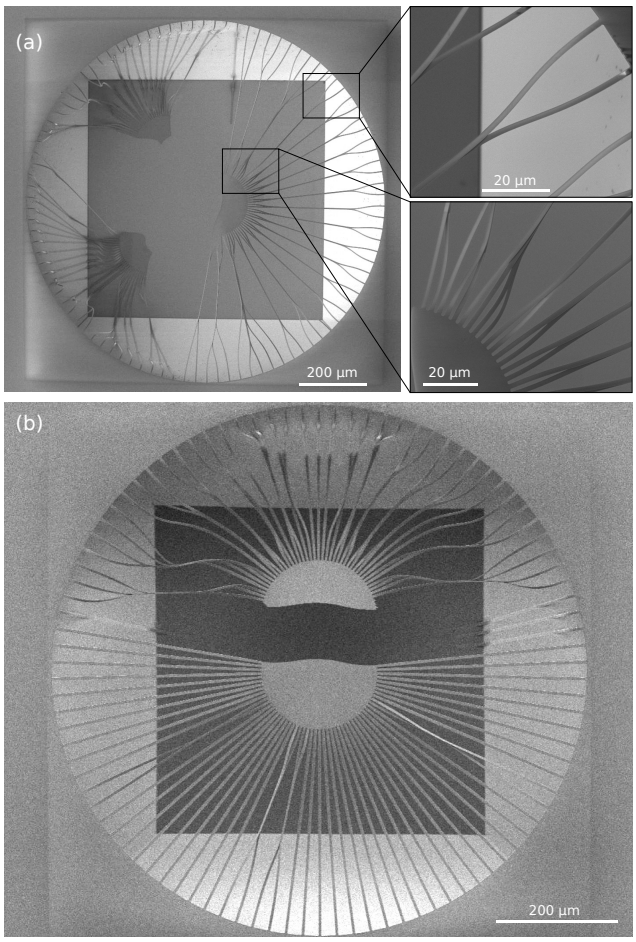

Figure S4 "Bicycle wheel" configuration with thin ribbons $(t=270 \mathrm{~nm})$. Although the wheels broke during the etching procedure, assemblies of ribbons are observed. Some particular assemblies twist as in the case of the isolated pairs described in the article. However most of the ribbons have here adopted an overlap configuration.

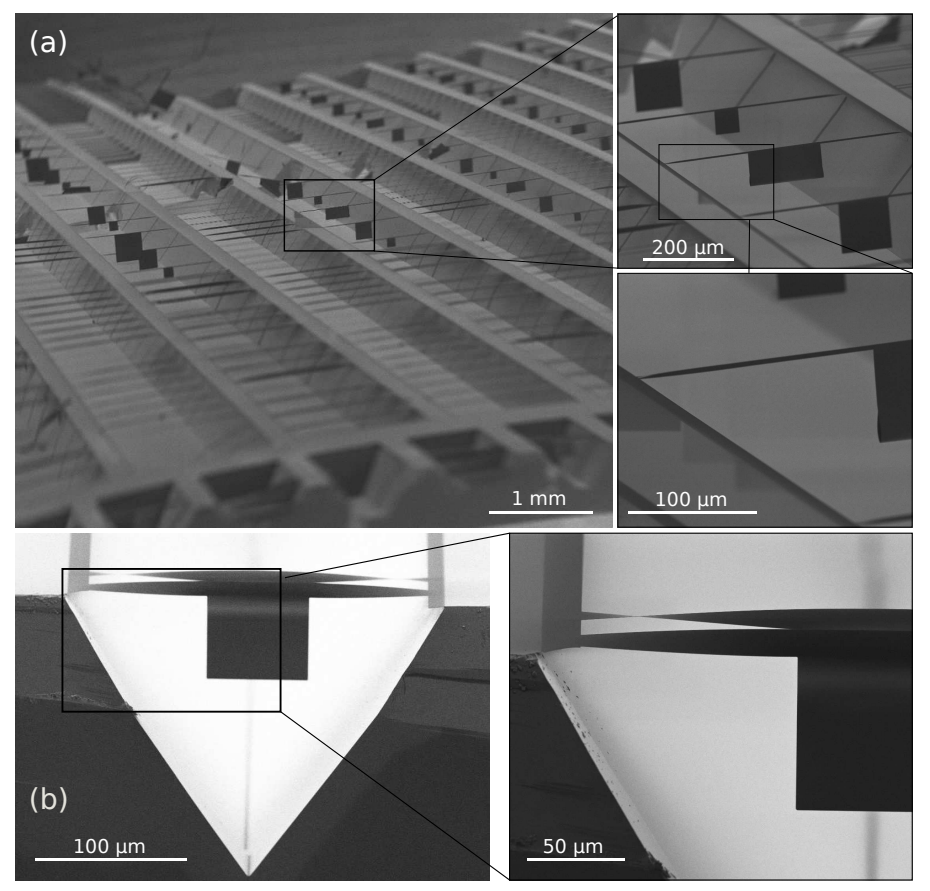

Figure S5 Assembly of ribbons with flaps on their sides. (a): Overview of structures with different geometries and close-up on a particular specimen. In this case the beams stick, as well as most of the flaps . (b): Side view of a shorter similar structure. The ribbons are only $L=200 \mu \mathrm{m}$ and would not assemble without the flaps. Displacement of the beams is negligible, while the twist angle is close to $90^{\circ}$. 


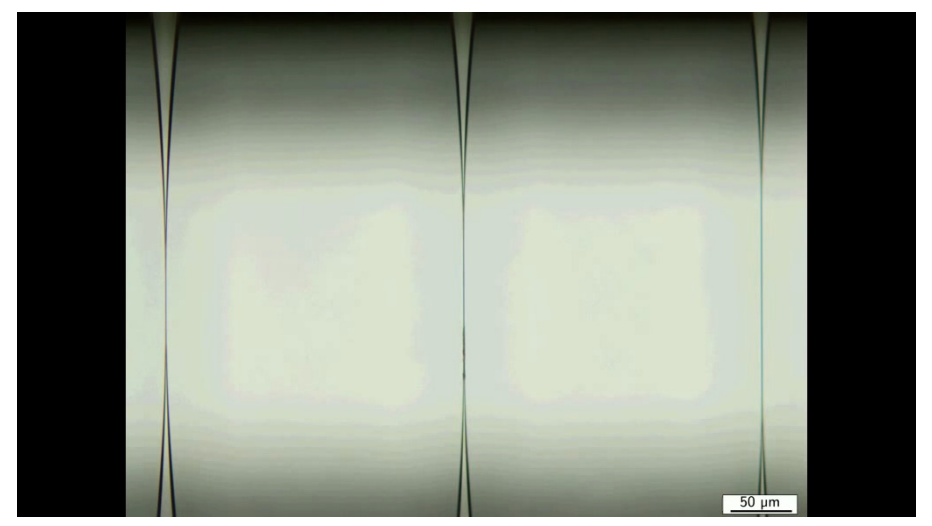

Movie 1. Experiment conducted with an array of micro-ribbons viewed by optical microscopy. As the water evaporates, capillary bridges bring the ribbons together. Note that the adhesion is maintained after the evaporation of water. This "dry" adhesion may be provided by traces of solutes present in the water and that may play the role of glue. 